Inversion of pore size dependence of solute transport kinetics from increasingly attractive ordered porous matrix

This content has been downloaded from IOPscience. Please scroll down to see the full text. 2010 EPL 9260011

(http://iopscience.iop.org/0295-5075/92/6/60011)

View the table of contents for this issue, or go to the journal homepage for more

Download details:

IP Address: 134.76.223.157

This content was downloaded on $30 / 08 / 2017$ at 14:35

Please note that terms and conditions apply.

You may also be interested in:

Enhancement of diffusive transport by non-equilibrium thermal fluctuations

Aleksandar Donev, John B Bell, Anton de la Fuente et al.

Effects of confinement on freezing and melting

C Alba-Simionesco, B Coasne, G Dosseh et al.

Water in nanopores: II. The liquid-vapour phase transition near hydrophobicsurfaces

Ivan Brovchenko, Alfons Geiger and Alla Oleinikova

Nonlinear rheology of colloidal dispersions

J M Brader

Solvation dynamics of molecular glass-forming liquids in confinement

Ranko Richert and Min Yang

Radiation Calculations with ZEUS-2D

N. J. Turner and J. M. Stone

Phase separation in confined systems

Lev D Gelb, K E Gubbins, R Radhakrishnan et al.

Thermal non-equilibrium transport in colloids

Alois Würger

Glass transition of polymers in bulk, confined geometries, and near interfaces

Simone Napolitano, Emmanouil Glynos and Nicholas B Tito 


\title{
Inversion of pore size dependence of solute transport kinetics from increasingly attractive ordered porous matrix
}

\author{
A. Godec ${ }^{(a)}$, T. Ukmar, M. Gaberščé and F. Merzel \\ National Institute of Chemistry - Hajdrihova 19, 1000 Ljubljana, Slovenia, EU
}

received 23 September 2010; accepted in final form 30 November 2010

published online 21 January 2011

PACS 02.60.Lj - Ordinary and partial differential equations; boundary value problems

PACS 05.60. Cd - Classical transport

PACS 47.63.mh - Transport processes and drug delivery

\begin{abstract}
The problem of solute transport in interacting ordered porous media is addressed by numerically solving the 2D Fokker-Planck equation using 4-step operator splitting. The subtle interplay between drift and diffusion is shown to result in a nontrivial dependence of solute transport kinetics on pore size. Depending on the strength of attraction to pore walls distinct regimes of pore size dependence of transport kinetics are found. The results suggest a decoupling of local dynamics from large-scale transport.
\end{abstract}

Copyright (C) EPLA, 2010

Introduction. - The importance of understanding molecular transport in porous media has long been recognized [1-6] and its applications range from rheology, composite materials, polymer and colloidal science, geology to biophysics and drug delivery. Intensive theoretical investigations of microscopic dynamics of fluids in porous media in the absence of large-scale concentration gradients have shed light on several subtle features of transport in random porous media, such as the interplay between self and collective dynamics in dilute and dense matrices [7], the qualitative transitions of particle dynamics depending on the obstacle density and configurations [8], distinct dynamical arrest transitions in glass-forming mixtures [9], long-time correlations due to quenched disorder [10] and breakdown of continuum hydrodynamics at the nanoscale [11]. Also it was shown, that the local dynamics are sensitive to the matrix structure (i.e. order) [12]. Moreover, the particle dynamics involving several time and length scales are very difficult to analyze. One therefore seeks an effective equation describing the particle behavior at long times and large scales. This can be done using homogenization theory [13]. It was shown, that the effective diffusivity in random or periodic velocity fields is always enhanced (compared to bare molecular diffusion) for incompressible flows $[14,15]$ and always depleted for potential flows [16-18]. Effective transport coefficients in external fields can also be obtained within the framework of linear response theory [19], for

\footnotetext{
(a) E-mail: aljaz.godec@ki.si
}

weak external fields, or nonlinear response [20], for fields of arbitrary magnitude. However, the local and large-scale dynamics in interacting ordered porous matrices in the presence of macroscopic concentration gradients are not well understood.

Here we address this problem by numerically solving the Fokker-Planck equation (FPE). We probe the influence of pore size and interaction strength and range on the transport kinetics within and from the matrix and address the influence of the initial degree of local concentration relaxation in the matrix. We also compute effective large-scale diffusion coefficients at long times and the evolution of the macroscopic concentration gradient for solute transport from the matrix for different pore sizes and attractions. We consider a simple 2-dimensional ordered porous model system (see fig. 1), which should be interesting for various applications, such as transport of reactants in membranes, nutrients or contaminants in soil, etc., but is particularly motivated by drug delivery from ordered mesoporous silicates.

By considering the dimension of the unit cell with respect to $L$ one immediately recognizes that a scale separation in the case of larger pores might not be achieved and that in turn the large-scale transport is also influenced by finite-size effects. Although a quantification of scale separation can only be done a posteriori, one can assume that in the case of larger pores it is certainly not achieved. However, it was shown [21] that in case of free diffusion in a system with a $2 \mathrm{D}$ period, which possesses two plane symmetries (which is the case in the present system), scale 




Fig. 1: Schematics of 2D porous matrix: $d$ denotes the pore size, $L$ the size of the porous matrix, $L_{B}$ denotes the buffer zone and $a$ the thickness of the pore wall. The gray area represents the pore volume. In all considered cases parameters $a$ and $d$ were chosen to assure equal pore volume, $V_{p}=L^{2}-n^{2} a^{2}=$ const., given the constant pore entrance area, $4(n-1) d=$ const., and constant particle size, $L=n a+(n-1) d$. Specifically we take $L=90 \Delta, L_{B}=155 \Delta$ and the following $d(n)$ values: $d_{0}(2)$, $d_{0} / 2(3), d_{0} / 3(4)$ and $d_{0} / 7(8)$, with $d_{0}=42 \Delta$. The grid origin is placed in the center of the matrix.

separation is not indispensable to obtain a large scale effective transport, that is independent of the total size of the porous matrix. Therefore, since the the external potential also fulfills the symmetry requirements, even in the case of largest pore size considered here the results in principle should not reflect any finite-size effects.

A 4-step operator splitting method [22] is used to solve the dynamical equations under reflecting boundary conditions. The size of the system is chosen to be large enough to assure that interactions vanish at large distances and that concentrations never reach appreciable values at external borders. The approach enables us to efficiently and systematically investigate the subtle interplay between energetic and entropic driving forces involved in solute transport in attractive ordered porous media.

Mathematical model. - The dynamics of the dimensionless solute concentration field $\varphi(\mathbf{r})$ are assumed to be governed by drift due to the local forces exerted on diffusing molecules by pore walls and diffusion due to the presence of local concentration gradients. Assuming a low Reynolds number environment and isotropic mobility, we model the system dynamics with the linear 2D macroscopic FPE

$$
\frac{\partial \varphi(\mathbf{r})}{\partial t}=D \nabla_{\mathbf{x y}} \cdot\left(\frac{\varphi(\mathbf{r})}{k_{B} T} \nabla_{\mathbf{x y}} V(\mathbf{r})+\nabla_{\mathbf{x y}} \varphi(\mathbf{r})\right) \equiv-\nabla_{\mathbf{x y}} \cdot \mathbf{j}
$$

where $\nabla_{\mathbf{x y}}$ represents the $2 \mathrm{D}$ gradient operator, $\nabla_{\mathbf{x y}}=$ $\partial / \partial x \hat{\mathbf{x}}+\partial / \partial y \hat{\mathbf{y}}, D$ is the diffusion coefficient, $k_{B}$ is the Boltzmann constant, $T$ is the temperature, $\mathbf{r}$ is the $2 \mathrm{D}$ position vector $\mathbf{r}=(x, y), V(\mathbf{r})$ represents the external potential field and $\mathbf{j}$ the generalized solute flux. We assume that the diffusing molecules interact with matrix wall molecules via pairwise additive potential of the Morse type,

$$
V(\mathbf{r})=q_{\min } \int_{R} \rho\left(\mathbf{r}^{\prime}\right)\left(e^{2 \sigma\left(d_{m}-\left|\mathbf{r}-\mathbf{r}^{\prime}\right|\right)}-2 e^{\sigma\left(d_{m}-\left|\mathbf{r}-\mathbf{r}^{\prime}\right|\right)}\right) \mathrm{d}^{2} \mathbf{r}^{\prime},
$$

where $\rho\left(\mathbf{r}^{\prime}\right)$ is the local density of the porous material $\left(\rho\left(\mathbf{r}^{\prime}\right)=1\right.$ if $\mathbf{r}^{\prime}$ lies within the wall and 0 otherwise), $q_{\text {min }}$ and $d_{m}$ are the depth and position of the pair potential minimum and $\sigma$ is the width of the potential well.

Numerical algorithm. - We discretize the FPE with 4-step operator splitting (see for example $[22,23]$ ): $\partial \varphi / \partial t=D \nabla_{\mathbf{x y}} \cdot\left(\varphi \nabla_{\mathbf{x y}} V(\mathbf{r}) / k_{B} T\right)+D \nabla_{x y}^{2} \varphi \equiv\left(\hat{\mathcal{L}}_{x}^{1}+\hat{\mathcal{L}}_{y}^{1}\right) \varphi+$ $\left(\hat{\mathcal{L}}_{x}^{2}+\hat{\mathcal{L}}_{y}^{2}\right) \varphi$, where the first and second pair of steps are treated with alternating-direction implicit method (ADI) and thus all substeps are differenced implicitly:

$$
\begin{aligned}
& \hat{\mathcal{L}}_{x}^{1} \rightarrow \frac{D}{2}\left(\frac{\varphi_{i+1, j}^{n+1 / 4}-2 \varphi_{i, j}^{n+1 / 4}+\varphi_{i-1, j}^{n+1 / 4}}{\Delta^{2}}\right. \\
& \left.+\frac{\varphi_{i, j+1}^{n}-2 \varphi_{i, j}^{n}+\varphi_{i, j-1}^{n}}{\Delta^{2}}\right), \\
& \hat{\mathcal{L}}_{y}^{1} \rightarrow \frac{D}{2}\left(\frac{\varphi_{i+1, j}^{n+1 / 4}-2 \varphi_{i, j}^{n+1 / 4}+\varphi_{i-1, j}^{n+1 / 4}}{\Delta^{2}}\right. \\
& \left.+\frac{\varphi_{i, j+1}^{n+1 / 2}-2 \varphi_{i, j}^{n+1 / 2}+\varphi_{i, j-1}^{n+1 / 2}}{\Delta^{2}}\right), \\
& \hat{\mathcal{L}}_{x}^{2} \rightarrow \frac{D}{2}\left(\frac{\varphi_{i+1 / 2, j}^{n+3 / 4} \delta \tilde{V}_{i+1 / 2, j}^{x}-\varphi_{i-1 / 2, j}^{n+3 / 4} \delta \tilde{V}_{i-1 / 2, j}^{x}}{\Delta^{2}}\right. \\
& \left.+\frac{\varphi_{i, j+1 / 2}^{n+1 / 2} \delta \widetilde{V}_{i, j+1 / 2}^{y}-\varphi_{i, j-1 / 2}^{n+1 / 2} \delta \widetilde{V}_{i, j-1 / 2}^{y}}{\Delta^{2}}\right), \\
& \hat{\mathcal{L}}_{y}^{2} \rightarrow \frac{D}{2}\left(\frac{\varphi_{i+1 / 2, j}^{n+3 / 4} \delta \tilde{V}_{i+1 / 2, j}^{x}-\varphi_{i-1 / 2, j}^{n+3 / 4} \delta \widetilde{V}_{i-1 / 2, j}^{x}}{\Delta^{2}}\right. \\
& \left.+\frac{\varphi_{i, j+1 / 2}^{n+1} \delta \widetilde{V}_{i, j+1 / 2}^{y}-\varphi_{i, j-1 / 2}^{n+1} \delta \widetilde{V}_{i, j-1 / 2}^{y}}{\Delta^{2}}\right),
\end{aligned}
$$

where $i$ and $j$ represent spatial coordinates, the superscript $n$ denotes the time step, $\varphi_{i \pm 1 / 2, j}^{n}=\left(\varphi_{i \pm 1, j}^{n}+\varphi_{i, j}^{n}\right) / 2$ and $\delta \widetilde{V}_{i, j-1 / 2}^{y}=\left(0.5\left[\widetilde{V}_{i, j+1}-\widetilde{V}_{i, j-1}\right]+0.5\left[\widetilde{V}_{i, j}-\widetilde{V}_{i, j-2}\right]\right) / 2$ are concentrations and components of external forces at mesh half-points, $\Delta x=\Delta y=\Delta$ is the grid spacing and $\widetilde{V}_{i, j}=V_{i, j} / k_{B} T$. Differencing of the potential at halfpoints is crucial for the numerical stability. Introducing $\alpha \equiv D \Delta t / \Delta^{2}$ and performing some basic algebraic manipulation eqs. (3)-(6) yield tridiagonal sets of coupled linear equations (for details see appendix).

The parameter $d_{m}$ in the pair potential is set equal to the grid spacing $\Delta$, which means that we consider only the attractive branch of the interaction. Hard repulsive interactions are introduced implicitly through Neumann boundary conditions for the concentrations and the potential. This way, there is no driving force for transport into pore walls and outer borders, as the drift $D \varphi \nabla_{\mathbf{x y}} V / k_{B} T$ 


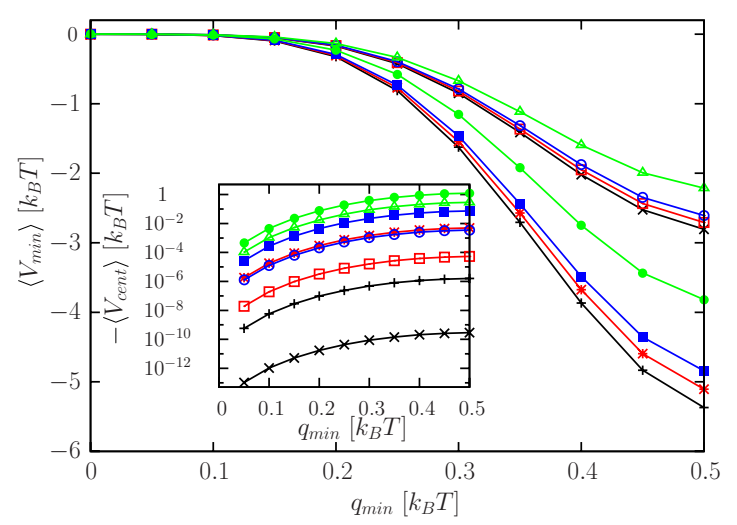

Fig. 2: $\left\langle V_{\text {min }}\right\rangle$ and $-\left\langle V_{\text {cent }}\right\rangle$ as a function of $q_{\text {min }}$ for all pore sizes considered in the calculations. Systems with $\sigma=0.8$ : $(\bullet)$ $d_{0} / 7,(\square) d_{0} / 3,(*) d_{0} / 2,(+) d_{0}$. Systems with $\sigma=1.2:(\triangle)$ $d_{0} / 7,(\circ) d_{0} / 3,(\square) d_{0} / 2,(\times) d_{0}$.

is also identically zero. Inside the walls the concentration is zero at all times. The resulting systems of equations are solved sequentially with LU decomposition, forward and backward substitution. All together we perform $1.2 \times 10^{5}$ time steps choosing $\alpha=0.2$.

External potential. - To establish a direct correspondence between the parameters of the pair potential and the resulting external potential we introduce the average potential in the center of the pores, $\left\langle V_{\text {cent }}\right\rangle$, and the average contact potential $\left\langle V_{\text {min }}\right\rangle$ (see footnote ${ }^{1}$ ), which is the potential averaged over all grid points that are adjacent to pore walls. In our calculations we consider two values for the pair potential width $\sigma$ (0.8 and 1.2) and potential depth $q_{\min }$ values ranging from 0 to 0.5 . Such choice of $\sigma$ is assumed to model realistic long- and shortranged pair attractions of different strengths. We consider 4 different porous systems with equal total pore volume as well as with equal total pore entrance area (for details see schematics in fig. 1). The dependence of the external potential on pore size (and thereby also wall thickness) expressed as $\left\langle V_{\text {min }}\right\rangle$ and $\left\langle V_{\text {cent }}\right\rangle$ as a function of $q_{\text {min }}$ is shown in fig. 2. On switching between $\sigma=0.8$ and $\sigma=1.2$ at given $q_{\min }$ and pore size, $\left\langle V_{\min }\right\rangle$ reduces on average by a factor of 2 , while $\left\langle V_{\text {cent }}\right\rangle$ is reduced by up to several orders of magnitude. For constant $\sigma$ the average contact attraction is strongest in case of larger pores and decreases monotonically with decreasing pore size, which follows directly from the corresponding wall thickness. The threshold where $\left\langle V_{\min }\right\rangle$ exceeds the thermal energy typically lies between $q_{\min }=0.27$ and $q_{\min }=0.5$, depending on $\sigma$ and pore size. $\left\langle V_{\text {cent }}\right\rangle$ becomes comparable to the thermal energy only in case of $d_{0} / 7$ for $q_{\min }>0.3$. From this observation we see, that the steady-state solution of

\footnotetext{
${ }^{1}$ We define the average contact potential as $\left\langle V_{\min }\right\rangle=\sum_{k=1}^{N_{w}}$ $\iint \mathrm{d}^{2} \mathbf{r} \mathrm{d} \ell \delta\left(\left|\mathbf{r}-\mathbf{r}_{w}^{k}(\ell)\right|-\Delta\right)(1-\rho(\mathbf{r})) V(\mathbf{r}) /\left[\sum_{k=1}^{N_{w}} \iint \mathrm{d}^{2} \mathbf{r} \mathrm{d} \ell \delta(\mid \mathbf{r}-\right.$ $\left.\left.\mathbf{r}_{w}^{k}(\ell) \mid-\Delta\right)(1-\rho(\mathbf{r}))\right]$, where $\mathbf{r}_{w}^{k}(\ell)=\left(x_{w}^{k}(\ell), y_{w}^{k}(\ell)\right)$ is the position vector of the $k$-th wall facet along its contour $\ell$ and the sum runs over all wall facets, $N_{w}$.
}

the FPE (eq. (1)), which is proportional to the Boltzmann distribution $\exp \left(-V(\mathbf{r}) / k_{B} T\right)$, will depart from a homogeneous concentration distribution mainly in the vicinity of pore walls (except for the smallest pore size).

Time evolution of the concentration field. - We consider two types of initial concentration distributions, a homogeneous distribution, where the solute concentration equals 1 inside the pores and zero outside, and a concentration proportional to the Boltzmann distribution inside the pores and zero outside. Initial distributions are normalized to assure equal total amount of solute in all cases. With this choice of initial conditions we can readily asses the relative timescales of the local and macroscopic solute concentration relaxation processes. In order to asses the influence of pore size on transport from interacting porous matrices we introduce the release half-time, $t_{1 / 2}$, which expresses the time at which one-half of the solute molecules have left the porous matrix. We define it implicitly as an integral of the total flux across the outer matrix surface:

$$
\int_{0}^{t_{1 / 2}} \mathrm{~d} \tau \oint_{O} \mathbf{j}(\mathbf{r}, \tau) \cdot \mathrm{d} \mathbf{S}=\frac{1}{2} \int_{R} \mathrm{~d}^{2} \mathbf{r} \varphi(\mathbf{r}, \tau=0) .
$$

$t_{1 / 2}$ is a measurable quantity which is also directly related to drug release experiments. We further assume that the large-scale solute transport can at long times be described by a 1D free-diffusion process with an effectivediffusion coefficient, $\partial \varphi_{l s} / \partial t=D_{e f f} \partial^{2} \varphi_{l s} / \partial x^{2} \quad$ which evolves from a box-shaped initial distribution with size $L$. In this case the solution is $\varphi_{l s}(x, t)=\varphi_{l s}^{0}(\operatorname{erf}[(L / 2-$ $\left.\left.x) /\left(2\left(D_{\text {eff }} t\right)^{1 / 2}\right)\right]+\operatorname{erf}\left[(L / 2+x) /\left(2\left(D_{\text {eff }} t\right)^{1 / 2}\right)\right]\right) / 2$ and the cumulative amount of solute outside the box (i.e. $|x|>L / 2)$ is $L \varphi_{l s}^{0}-\int_{-L / 2}^{L / 2} \varphi_{l s} \mathrm{~d} x$. The fraction of solute outside the matrix as a function of time is found by performing the integral explicitly and can be shown to be given by

$$
\Theta(t)=1-\frac{\operatorname{erf}\left[\frac{L}{2 \sqrt{D_{e f f} t}}\right]}{4 D_{e f f} t}-\frac{\exp \left[\frac{-L^{2}}{4 D_{e f f} t}\right]-1}{2 L \sqrt{\pi D_{e f f} t}} .
$$

$D_{\text {eff }}$ is in turn obtained from $\varphi(\mathbf{r}, t)$ by fitting $\int_{0}^{t} \mathrm{~d} \tau \oint_{O} \mathbf{j}(\mathbf{r}, \tau) \cdot \mathrm{d} \mathbf{S}$ with eq. (8) for long times. The last $25 \times 10^{4}$ steps were used in the fitting procedure and the largest standard deviation in obtained $D_{\text {eff }}$ was below $0.1 \%$. As shown in figs. $3(\mathrm{a})$ and (c) reduction of pore size results in faster transport from the matrix for $q_{\min }<0.25$ in case of $\sigma=1.2\left(q_{\min }<0.2\right.$ in case of $\sigma=0.8$, respectively). This was also demonstrated in the case of nonlinear diffusion (i.e. assuming a concentration-dependent diffusion coefficient) in porous media with hard repulsive walls [24]. On the other hand, a reduction of pore size always slows down transport from the matrix in the case of $q_{\min }>0.4$ (for $\sigma=1.2$ ) and $q_{\min }>0.35$ (for $\sigma=0.8$ ). For intermediate values of $q_{\min }$ a reduction of pore size may in fact enhance or slow down solute transport from the matrix, depending 


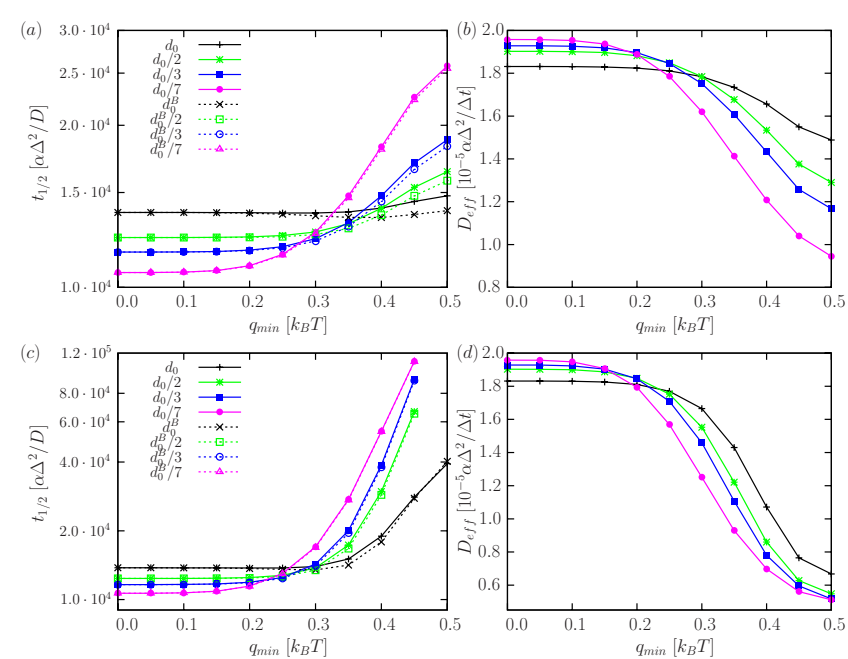

Fig. 3: $t_{1 / 2}$ in the case of (a) $\sigma=1.2$ and (c) $\sigma=0.8$ as calculated using eq. (7) (broken lines denote $t_{1 / 2}$ in the case of Boltzmann-distributed initial concentration within the matrix). After solving for $\varphi(\mathbf{r}, \tau)$ the integral was evaluated numerically. Long-time effective-diffusion coefficients in the case of (b) $\sigma=1.2$ and (d) $\sigma=0.8$. $D_{\text {eff }}$ for the initially Boltzmann-distributed concentration are not shown because they do not differ from the flat initial distribution (the difference is within the error).

on the pore size. In this mixed regime, $\left\langle V_{\min }\right\rangle \approx-k_{B} T$, while $\left\langle V_{\text {cent }}\right\rangle$ is (except in the case of smallest pore size) negligible. In case of increasingly stronger attractions, drift always dominates and a reduction of pore size slows down transport from the matrix. Except in the case of largest pore size, $t_{1 / 2}$ is essentially independent of initial conditions and thus the degree of initial local relaxation, which already suggests that the microscopic relaxation is much faster. Note that for the largest pore size $t_{1 / 2}$ in fact decreases upon increasing attraction if starting from the Boltzmann distribution, which suggest that the drift-dominated local relaxation becomes slower in case of larger pores. Consistent with $t_{1 / 2}$ behavior, the pore size dependence of $D_{\text {eff }}$ (figs. 3(c) and (d)) also becomes inverted in the case of sufficiently strong attractions. In the absence of strong interactions, the entropic driving force (concentration gradient) will completely dominate the local dynamics of the solute concentration, except in the immediate proximity of pore walls, where we may expect some drift in the direction towards pore walls due to attractive forces. A snapshot of local fluxes at the border of the porous matrix for the $d_{0} / 7$ system with $q_{\text {min }}=0.2$ (just below the mixed regime) at $\tau=5 \cdot 10^{3} \Delta t$ $\left(\Delta t=\alpha \Delta^{2} / D\right)$ is shown in figs. 4(a) and (b). As observed, near the matrix surface the total flux is dominated by the entropic driving force, causing a significant flux from the matrix. Going towards the center of the matrix, the drift component gradually builds up until it completely dominates. The flux along the pore increases from the center of the matrix to its surface. Larger concentration gradients are maintained near the surface, which is even

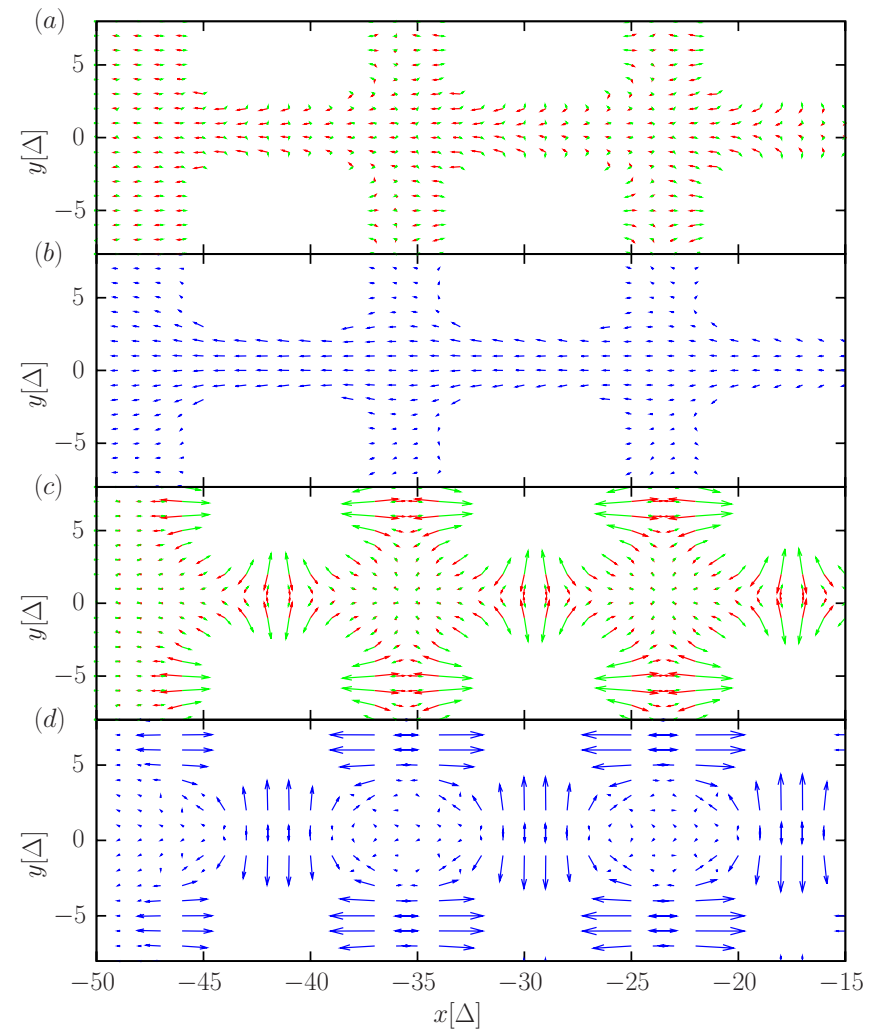

Fig. 4: Diffusive (red) and drift (green) components together with the total flux (blue arrows) in the porous matrix with smallest pore size at time $\tau=5 \cdot 10^{3} \Delta t$ for $\sigma=0.8$. (a) and (b) correspond to $q_{\min }=0.2 k_{B} T$ and (c) and (d) correspond to $q_{\min }=0.4 k_{B} T$. The vectors are scaled up for visual convenience (the ratio of scales (a) : (b) : (c) : (d) is $28: 20: 1: 5$ ). The outermost pore walls are located at $(-45, \pm 3)$; the position of the vectors corresponds to their origin.

more pronounced in the case of larger pores (see text below and concentration distributions for $q_{\min } \leqslant 0.2$ in fig. 5). In the presence of strong attractions drift dominates everywhere except in the center of the pores, where the force in the direction of the wall is zero by symmetry (see figs. 4(c) and (d) showing a snapshot for $d_{0} / 7$ and $q_{\min }=0.4$ at $\tau=5 \cdot 10^{3} \Delta t$ ). The flux from the porous matrix is strongly reduced. Note however, that the drift-dominated local dynamics inside the matrix are in fact very intense and the total local fluxes are significantly larger than in the case of weaker attractions. The net effect of local fluxes is to cause the solute to accumulate near the walls and to establishes, what can be understood as, a sort of multilayer adsorption (see also fig. 5). Therefrom $\varphi(\mathbf{r})$ slowly relaxes towards to the Boltzmann distribution. The progressively stronger adsorption can be nicely observed from the growing contact concentration in the pore. By comparing figs. 5(a) and (b), which show concentration profiles through the matrix for two different pore sizes, we observe, that the total solute concentration difference between the center of the porous matrix and the surrounding medium is significantly larger in the case of smaller pores. If we 

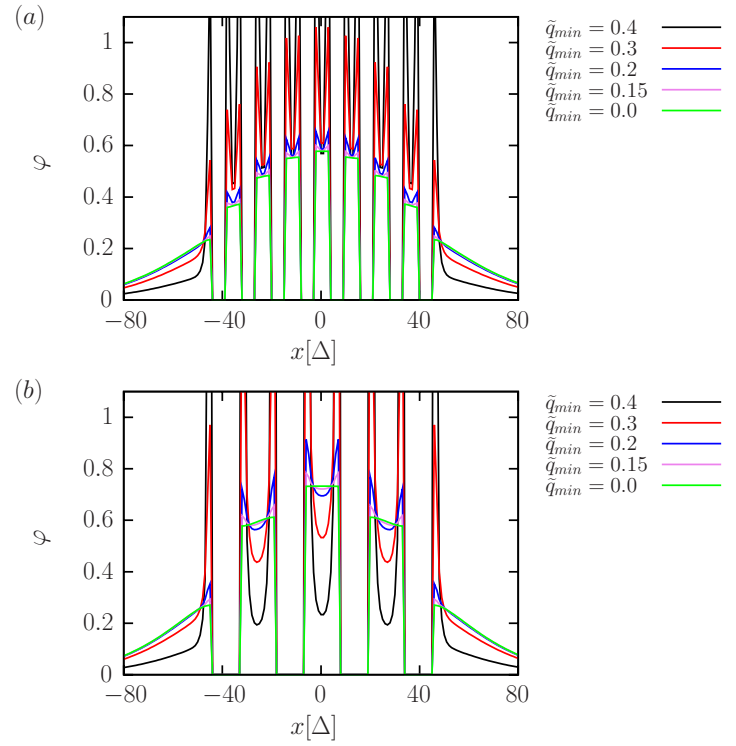

Fig. 5: Concentration distribution at time $\tau=10^{4} \Delta t$ for $\sigma=$ 0.8 and several values of $q_{\min }$ : (a) the $d_{0} / 7$ porous system at $y=-8 \Delta$ along the $x$-direction and (b) the $d_{0} / 3$ porous system at $y=-12 \Delta$ along the $x$-direction. Regions with zero concentration correspond to pore walls.

define the average concentration in the center of the matrix, $\overline{c_{i n}}$, as the average over points lying in the center of pores of the smallest central symmetric unit and the average external concentration, $\overline{c_{\text {out }}}$, as the average over the square with side $L+10 \Delta$, we can quantify the effective macroscopic driving force in terms of the average concentration difference, $\overline{\Delta c}=\overline{c_{\text {in }}}-\overline{c_{\text {out }}}$. The time evolution of $\overline{\Delta c}$ is shown in fig. 6. By comparing the full and broken lines one readily observes the influence of initial conditions on the overall driving force for large-scale solute transport. As expected the initial $\overline{\Delta c}$ difference for Boltzmann-distributed and flat initial conditions is larger in the case of stronger and long-ranged attractions, while at given interaction it is larger in the case of smaller pores. The time at which both curves, corresponding to a given $q_{\text {min }}, \sigma$ and $d$, converge may be interpreted as the local microscopic relaxation timescale. This timescale is obviously pore size dependent and is longer in the case of larger pores, which follows directly from the distance which solute molecules have to traverse prior to adsorption at the pore wall if starting from a flat distribution. More interestingly it appears to be almost independent of $q_{\min }$ and $\sigma$ at a given $d$, which means the the entropic and energetic effects on large-scale transport balance each other upon increasing attraction strength and range at a given pore size. In general the microscopic relaxation is up to 3 orders of magnitude faster than the macroscopic solute transport as measured with $t_{1 / 2}$. This explains the apparent independence of $t_{1 / 2}$ from the initial concentration distribution. From fig. 6 one also observes that the effective macroscopic concentration gradient at long times is larger for smaller
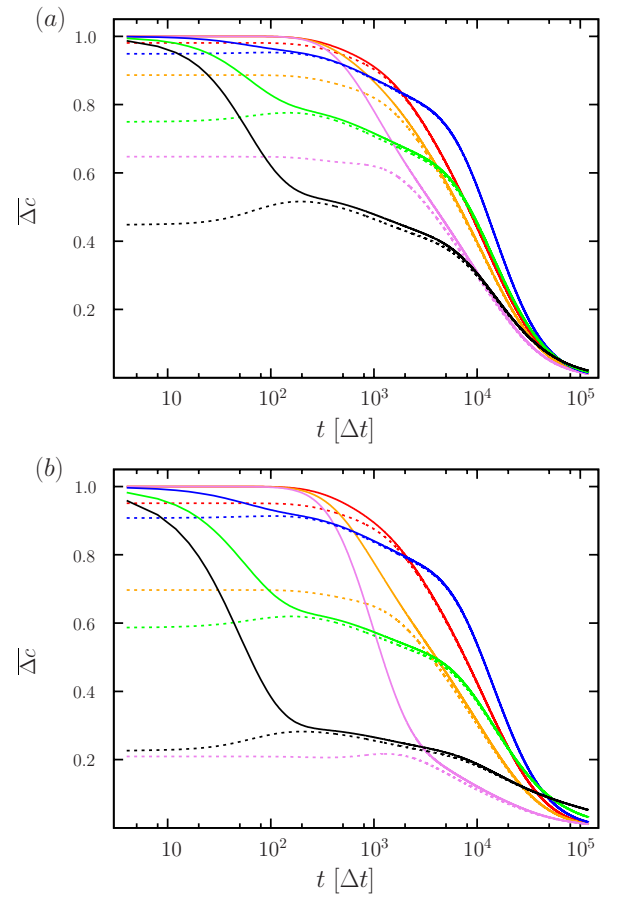

Fig. 6: Time evolution of $\overline{\Delta c}$ in the case of (a) $\sigma=1.2$ and (b) $\sigma=0.8$. Results for the $d_{0} / 3$ porous system: (-) $q_{\text {min }}=0.2,(-) q_{\min }=0.3,(-) q_{\min }=0.4 ;$ results for the $d_{0} / 7$ porous system: $(-) q_{\min }=0.2,(-) q_{\min }=0.3,(-) q_{\min }=0.4$. Broken lines correspond to the initially Boltzmann distributed and full lines to the homogeneously distributed concentration inside the pores.

pores. Hence, the overall entropic driving force, is larger due to a more attractive potential in the center of smaller pores. The relative cross-section of the pore effectively contributing to transport along the pore, which decisively contributes to $D_{\text {eff }}$ in figs. $3(\mathrm{~b})$ and (d), is larger in the case of larger pores. The total entropic driving force, however, is smaller. Hence we see, that although an inversion of transport kinetics form the porous matrix occurs at sufficiently strong attraction regardless of the pore size, the underlying mechanism differs in smaller and larger pores. Even though it is always mediated by drift, reduction of kinetics in smaller pores occurs due to a smaller effective-axial-transport region (manifested macroscopically as a lower $D_{\text {eff }}$ ) and a more favorable attractive potential in the center of the pores, while in larger pores the total entropic driving force is strongly reduced due to more extensive adsorption (and in turn larger depletion in the region of effective axial transport and thus smaller $\overline{\Delta c}$ ). By comparing concentration distributions in fig. 5 , for $q_{\text {min }} \leqslant 0.2$ effects of external forces are strictly local and affect the concentration only inside single pores (which is seen in fig. 3), while upon increasing strength of interaction the coupling of local effects gradually increases.

Our results also suggest that the generally accepted and experimentally confirmed effect of reduction of drug release kinetics upon reduction of pore size [25-28] can 
only be reproduced theoretically by including sufficiently strong attractions to pore walls.

One important issue, which has not been addressed here, is also the effect of transport acceleration by reduction of dimensionality [29], where the geometry of the external potential creates a preferential connected pathway through the medium, which can accelerate solute transport as compared to the absence of potential. Such a study is currently in progress.

Summary. - In this letter we addressed a fundamental issue of solute transport in attractive ordered porous media, in particular the effect of pore size and solute-wall attraction. We found that the pore size dependence of solute transport kinetics from the porous matrix greatly depends on the strength and range of attractions to pore walls. Regardless of pore size, solute transport from the matrix is always reduced in case of sufficiently strong attractions to pore walls. The mechanism leading to this depletion of release, however, differs in smaller and larger pores. In the first case the dominant mechanism appears to be a reduction of the effective large-scale diffusion coefficient and in the latter (especially for short-range attractions) a reduction of the entropic driving force for largescale transport. We found the local dynamics to be rather decoupled from the large-scale transport. As a result, the overall solute release kinetics are insensitive to the degree of initial local relaxation. The local relaxation timescale depends on the pore size, but is, rather surprisingly, insensitive to the strength and range of attractions to pore walls. Our findings may impact the design of novel porous matrices, especially for application in drug delivery.

\section{APPENDIX}

Upon elementary algebraic manipulation of eqs. (3)-(6) one obtains tridiagonal sets of coupled linear equations $\mathrm{T}_{k}^{l} \boldsymbol{\varphi}=\mathbf{r}_{k}^{l}, k=x, y$ and $l=1,2$. The explicit forms of $\mathrm{T}_{k}^{l}$ and $\mathbf{r}_{k}^{l}$ corresponding to specific substeps are the following.

For the first two ADI substeps we have $\mathrm{T}_{x}^{1}=\mathrm{T}_{y}^{1} \equiv \mathrm{T}^{1}$, with diagonal elements equal to $1+\alpha$ and off-diagonal elements $-\alpha / 2$. The corresponding vector components are $r_{i, x}^{1}=\alpha \varphi_{i, j+1}^{n} / 2+(1-\alpha) \varphi_{i, j}^{n}+\alpha \varphi_{i, j-1}^{n} / 2$ and $r_{i, y}^{1}=$ $\alpha \varphi_{i+1, j}^{n+1 / 4} / 2+(1-\alpha) \varphi_{i, j}^{n+1 / 4}+\alpha \varphi_{i-1, j}^{n+1 / 4} / 2$ respectively. For the third substep the elements of $\mathrm{T}_{x}^{2}$ are $b_{i}^{x}$ (diagonal), $a_{i}^{x}$ (the left off-diagonals) and $c_{i}^{x}$ (the right off-diagonals), where $a_{i}^{x}=\alpha \delta \widetilde{V}_{i-1 / 2, j}^{x} / 4, b_{i}^{x}=1-\alpha\left(\delta \widetilde{V}_{i+1 / 2, j}^{x}-\delta \widetilde{V}_{i-1 / 2, j}^{x}\right) / 4$, $c_{i}^{x}=-\alpha \delta \widetilde{V}_{i+1 / 2, j}^{x} / 4$. The vector components are $r_{i, x}^{2}=$ $\alpha \delta \widetilde{V}_{i, j+1 / 2}^{y} \varphi_{i, j+1}^{n+1 / 2} / 4+\left(1+\alpha\left[\delta \tilde{V}_{i, j+1 / 2}^{y}-\delta \tilde{V}_{i, j-1 / 2}^{y}\right]\right) \varphi_{i, j}^{n+1 / 2}-$ $\alpha \delta \tilde{V}_{i, j-1 / 2}^{y} \varphi_{i, j-1}^{n+1 / 2} / 4$. For the last substep the elements of $\mathrm{T}_{y}^{2}$ are $b_{j}^{y}$ (diagonal), $a_{j}^{y}$ (the left off-diagonals) and $c_{j}^{y}$ (the right off-diagonals), with $a_{j}^{x}=\alpha \delta \tilde{V}_{i, j-1 / 2}^{x} / 4, \quad b_{j}^{y}=1-\alpha$ $\left(\delta \widetilde{V}_{i, j+1 / 2}^{y}-\delta \widetilde{V}_{i, j-1 / 2}^{y}\right) / 4, \quad c_{j}^{x}=-\alpha \delta \widetilde{V}_{i+1 / 2, j}^{x} / 4$ and the vector components $r_{j, y}^{2}=\alpha \delta \widetilde{V}_{i+1 / 2, j}^{x} \varphi_{i+1, j}^{n+3 / 4} / 4+(1+$ $\left.\alpha\left[\delta \widetilde{V}_{i+1 / 2, j}^{x}-\delta \widetilde{V}_{i-1 / 2, j}^{x}\right]\right) \varphi_{i, j}^{n+3 / 4}-\alpha \delta \widetilde{V}_{i-1 / 2, j}^{x} \varphi_{i-1, j}^{n+3 / 4} / 4$.

\section{REFERENCES}

[1] Stauffer D., Phys. Rep., 54 (1979) 1.

[2] Nakayama T., Yakubo K. and Orbach R. L., Rev. Mod. Phys., 66 (1994) 381.

[3] Höfling F., Franosch T. and Frey E., Phys. Rev. Lett., 96 (2006) 165901.

[4] Halperin B. I., Feng S. and Sen P. N., Phys. Rev. Lett., 54 (1985) 2391.

[5] Mair R. W., Wong G. P., Hoffmann D., Hürlimann M. D., Patz S., Schwartz L. M. and Walsworth R. L., Phys. Rev. Lett., 83 (1999) 3324.

[6] Berkowitz B., Klafter J., Metzler R. and Scher H., Water Resour. Res., 38 (2002) 1191.

[7] Kurzidim J., Coslovich D. and Kahl Gerhard, Phys. Rev. Lett., 103 (2009) 138303.

[8] Kim K., Miyazaki K. and Saito S., EPL, 88 (2009) 36002.

[9] Voigtmann Th. and Horbach J., Phys. Rev. Lett., 103 (2009) 205901.

[10] Franosch T., Höfling F., Bauer T. and Frey E., Chem. Phys., 375 (2010) 540.

[11] Russo J., Horbach J., Sciortino F. and Succi S., EPL, 89 (2010) 44001.

[12] Sung B. J. and Yethiraj A., J. Chem. Phys., 128 (2008) 054702.

[13] Bensoussan A., Lions J. L. and Papanicolaou G., Asymptotic Analysis of Periodic Structures (NorthHolland, Amsterdam) 1978.

[14] Fannjiang A. and Papanicolaou G. C., SiAM J. Appl. Math., 54 (1994) 333.

[15] Majda A. J. and Kramer P. R., Phys. Rep., 314 (1999) 237.

[16] Vergassola M. and Avellaneda M., Physica D, 1-2 (1997) 148.

[17] Rodenhausen H., J. Stat. Phys., 55 (1989) 1065.

[18] Garnier J., SIAM J. Appl. Math., 57 (1997) 95.

[19] Kubo R., Rep. Prog. Phys., 29 (1966) 255.

[20] Parris P. E., Kus M., Dunlap D. H. and Kenkre V. M., Phys. Rev. E, 56 (1997) 5295.

[21] Auriault J.-L. and Lewandowska J., Transp. Porous Media, 43 (2001) 473.

[22] Press W. H., Teukolsky S. A., Vetterling W. T. and Flannery B. P., Numerical Recipes: The Art of Scientific Computing, 3rd edition (Cambridge University Press, New York) 2007.

[23] Zorzano M. P., Mais H. and Vazquez L., Appl. Math. Comput., 98 (1999) 109.

[24] Godec A., Gaberscek M., Jamnik J. and Merzel F., J. Chem. Phys., 131 (2009) 234106.

[25] Vallet-Regí M., Balas F. and Arcos D., Angew. Chem. Int. Ed., 46 (2007) 7548.

[26] Wang S., Microporous Mesoporous Mater., 117 (2009) 1.

[27] Song S. W., Hidajat K. and Kawi S., Langmuir, 21 (2005) 9568.

[28] Doadrio J. C., Sousa E. M. B., Izquierdo-Barba I., Doadrio A. L., Perez-Pariente J. and Vallet-Regí M., J. Mater. Chem., 16 (2006) 462.

[29] Loverdo C., Benichou O., Moreau M. and Voituriez R., Nat. Phys., 4 (2008) 134. 